\title{
Determining the Effective Constitutive Parameters of Finite Periodic Structures: Photonic Crystals and Metamaterials
}

\author{
M. I. Aksun, Senior Member, IEEE, Aytaç Alparslan, Student Member, IEEE, E. P. Karabulut, Student Member, IEEE, \\ Erdinc Irci, Student Member, IEEE, and Vakur B. Ertürk, Member, IEEE
}

\begin{abstract}
A novel approach to find the effective electric and magnetic parameters of finite periodic structures is proposed. The method uses the reflection coefficients at the interface between a homogenous half-space and the periodic structure of different thicknesses. The reflection data are then approximated by complex exponentials, from which one can deduce the wavenumber, and the effective electric and magnetic properties of the equivalent structure by a simple comparison to the geometrical series representation of the generalized reflection from a homogenous slab. Since the effective parameters are for the homogenous equivalent of the periodic structure, the results obtained are expected to be independent of the number of unit cells used in the longitudinal direction. Although the proposed method is quite versatile and applicable to any finite periodic structure, photonic crystals and metamaterials with metallic inclusions have been used to demonstrate the application of the method in this paper.
\end{abstract}

Index Terms-Effective constitutive parameters, homogenization, metamaterials, photonic crystals.

\section{INTRODUCTION}

$\mathbf{R}$ ECENT development in periodic structures, such as photonic crystals and metamaterials, has created a flurry of interest among scientists from different fields, [1]-[4]. This excitement can be attributed mainly to their unique electromagnetic (EM)/optical features, and potentials for new and exciting applications, [5]-[7]. As these structures are still being studied to understand their basic operations, some applications in the fields of microwave circuits, antennas, and optics have already begun to appear in the literature. Since the common feature of such structures is their periodic nature, EM-based full-wave analysis tools, designed for or capable of handling periodic structures, play an important role to understand the underlying physical mechanisms, as well as to help designing such structures [8]-[12]. However, when it comes to building practical circuits in or on such structures, accurate simulation

Manuscript received August 15, 2007; revised February 2, 2008. This work was supported in part by TÜBİTAK under Contract 105E141, and in part by TÜBA-GEBİP.

M. I. Aksun, A. Alparslan, and E. P. Karabulut are with the Department of Electrical and Electronics Engineering, Koç University, 34450 Istanbul, Turkey (e-mail: iaksun@ku.edu.tr; aalparslan@ku.edu.tr; ekarabulut@ku.edu.tr).

E. Irci is with the ElectroScience Laboratory, The Ohio State University, Columbus, OH 43210 USA (e-mail: irci.1@osu.edu).

V. B. Ertürk is with the Department of Electrical and Electronics Engineering, Bilkent University, 06800 Bilkent, Ankara, Turkey (e-mail: vakur@ee.bilkent. edu.tr).

Digital Object Identifier 10.1109/TMTT.2008.923870 tools accounting for all the details may not be necessary for some applications. Instead, one may use an equivalent set of EM parameters, also referred to as effective parameters, to approximate the structure as a homogenous medium. In the literature, there are mainly three different techniques to obtain these effective parameters, which are, namely, static or quasi-static approaches [13], [14], averaging procedures [15]-[17], or retrieval procedures [18]-[21].

Parameter retrieval techniques for metamaterials, from a set of transmission and reflection coefficient data, have recently become quite popular, in order to characterize such structures, and to help design different configurations for negative effective permittivity and permeability values. However, this approach provides effective parameters based on the measurement or calculation of a fixed number of unit cells in the direction of propagation, which may not represent the effective parameters of an equivalent homogeneous structure with an arbitrary number of unit cells. In other words, interactions between the building blocks of the periodic structure may not be simply neglected if the intention is not to retrieve the effective parameters of the given configuration, but to determine the effective parameters of its homogeneous equivalent for an arbitrary number of unit cells. In this paper, a novel approach based on the reflection data, for a set of overall thickness of the structure, is proposed to find the effective permittivity and permeability values that could represent the structure regardless of the number of unit cells used. The method is capable of providing the effective parameters of any finite extent periodic structure, including those with defects or with inhomogeneous dielectric materials, provided that the reflection data can be obtained at the front interface of the structure. In addition, the method can work not only with normal incident plane waves, but with plane waves of any arbitrary angle of incidence. Since EM-based numerical simulation tools, like Comsol Multiphysics by Comsol Inc., Burlington, MA, and the High Frequency Structure Simulator (HFSS) by Ansoft Inc., Pittsburgh, PA, have become quite common and accessible, reflection coefficient data of any structure can easily be obtained from the field distributions.

Since the main contribution of this paper is to develop a general-purpose algorithm that provides the effective parameters of finite extent periodic structures, the proposed algorithm and its working mechanism are detailed in Section II. It is then followed by some representative examples, chosen mainly from 1-D and 2-D photonic crystals, and 2-D and 3-D metamaterials in Section III. Finally, conclusions are provided in Section IV. 


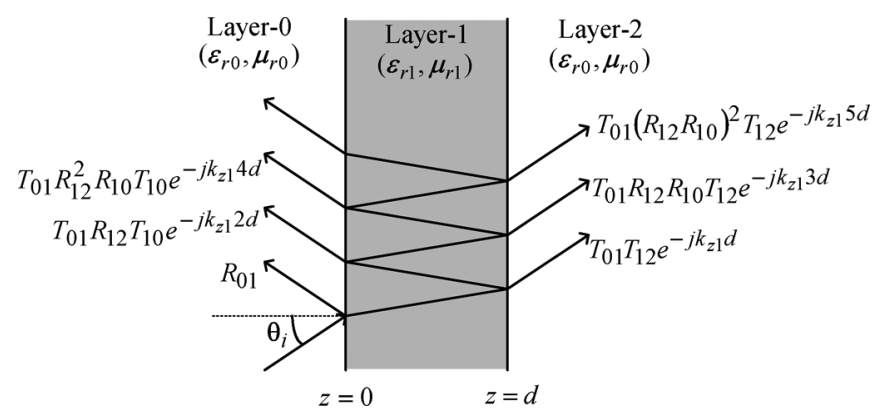

Fig. 1. Multiple reflections and transmissions from a homogenous slab.

\section{Details OF THE METHOD}

The proposed method starts with obtaining the reflection coefficient data for a different number of unit cells of the periodic structure. The data are then approximated by a sum of complex exponentials, and each exponential term in the approximation is assumed to be equal to a term in the geometric series representation of the total reflection from a homogenous slab. Hence, the wavenumber of the equivalent homogenous medium is obtained from the exponents of the approximating exponentials. Subsequently, the effective permittivity and permeability values can be obtained from the exponential term of the approximation that corresponds to the Fresnel reflection coefficient in the geometric series.

To understand the proposed method, it would be necessary to review the reflection of EM waves at the interface between a semiinfinite homogeneous medium $(z<0)$ and a homogenous slab $(0 \leq z \leq d)$, as shown in Fig. 1 . The reflection coefficient defined at the front interface $(z=0)$ can be written in terms of multiple reflections of the wave inside the slab as

$$
\begin{aligned}
\tilde{R}_{01}= & R_{01}+T_{01} T_{10} R_{12} e^{-j k_{z 1} 2 d}+ \\
& +T_{01} T_{10} R_{10} R_{12} e^{-j k_{z 1} 4 d}+\cdots \\
= & R_{01}+\frac{T_{01} T_{10} R_{12} e^{-j k_{z 1} 2 d}}{1-R_{12} R_{10} e^{-j k_{z 1} 2 d}}
\end{aligned}
$$

where $\sim$ over $R$ denotes the generalized reflection coefficient, accounting for multiple reflections, and $R$ and $T(=1+R)$ without $\sim$ are the Fresnel reflection and transmission coefficients, accounting just for the discontinuity of the two media [22]. In addition, the first and second subscripts give the layer numbers of the incident and transmitted media, respectively. Note that the geometric series is the result of the homogeneity of the slab, and that each exponential term is a function of the thickness and the wavenumber of the slab. Therefore, if the reflection coefficient, measured or obtained by simulations, of any structure demonstrates such a behavior at a given frequency, one can confidently represent the structure by an equivalent homogeneous medium at that frequency.

Although the generalized reflection coefficient expression (1) is the same for both TE and TM waves, the reflection and transmission coefficients in the expression are different for these waves. For example, the first term in the expansion $R_{01}$-referred to as Fresnel reflection coefficient-accounts for the difference between the media on both sides of the interface, and is defined for TE and TM waves as follows:

$$
\begin{aligned}
R_{01}{ }^{\mathrm{TE}} & =\frac{\mu_{1} k_{z 0}-\mu_{0} k_{z 1}}{\mu_{1} k_{z 0}+\mu_{0} k_{z 1}} \\
R_{01}{ }^{\mathrm{TM}} & =\frac{\varepsilon_{1} k_{z 0}-\varepsilon_{0} k_{z 1}}{\varepsilon_{1} k_{z 0}+\varepsilon_{0} k_{z 1}} .
\end{aligned}
$$

The nice thing about the Fresnel reflection coefficient is that it is just a result of discontinuity at the interface between two media, Layer-0 and Layer-1 in Fig. 1, independent of whatever the wave experiences afterwards. Therefore, it is only dependent on the propagation constant (in the normal direction to the plane of interface), permittivity and permeability of the two homogenous semiinfinite media.

Although the generalized reflection coefficient formula is valid for a homogenous slab with a fixed thickness $d$, it can be considered as a thickness-dependent equation, i.e., an equation that relates the change in thickness to the reflection coefficient seen at the front interface. Note that, throughout this study, the first and second effective boundaries of the equivalent slab are assumed to coincide with the first and last unit-cell boundaries of the finite periodic structure, respectively, as concluded in [20] for symmetric 1-D metamaterials. Since the unit cells defined for all the structures used in this study are symmetric, and because the metamaterials are only 1-D, this choice of the boundaries of the equivalent slabs can be justified. However, in cases of higher dimensional metamaterials and/or structures with asymmetric unit cells, the effective boundaries can be determined by the optimization algorithm presented in [20]. As a result, a set of reflection coefficient data collected at the front interface for different thicknesses can be approximated by a finite sum of exponentials as

$$
\tilde{R}_{01}(z=0, t) \cong \sum_{m=0}^{M-1} a_{m} e^{\alpha_{m} t}
$$

where the generalized pencil-of-function method is used for the exponential approximation [23], the coefficients $a_{m}$ and exponents $\alpha_{m}$ are complex numbers, and $t$ is the thickness of the equivalent slab. Note that the samples of the generalized reflection coefficient are collected at the integer multiples of the length of a unit cell $d$ with uniformly increasing thicknesses, starting from a certain bias thickness to eliminate the dominance of the interactions between the constituting structures of a unit cell. This sampling strategy can be best described by the following formula: $\tilde{R}_{01}(z=0, t=n d)$ for $n=N_{0}+1, \ldots, N_{0}+$ $N$, where $N_{0}$ is the initial number of unit cells.

As stated above, the motivation for the exponential approximation is that the resulting sum of exponentials should approximate the geometrical series representation of the generalized reflection coefficient term by term, provided the periodic structure can be represented by a homogeneous equivalent slab at the wavelength of operation. Therefore, forming a one-to-one correspondence between the series representation of the generalized reflection coefficient (1) and the approximating sum of exponentials (3), the following map between the coefficients and 
exponents can be constructed:

$$
\begin{array}{ll}
a_{0}=R_{01} & \alpha_{0}=0 \\
a_{1}=T_{01} T_{10} R_{12} & \alpha_{1}=-j 2 k_{z 1} \\
a_{2}=T_{01} T_{10} R_{10} R_{12}^{2} & \alpha_{2}=-j 4 k_{z 1}
\end{array}
$$

and, subsequently, the effective propagation constant in Layer-1, in the direction normal to the interface, can be obtained as

$$
\operatorname{Re}\left[k_{z 1}\right] d=-\frac{\operatorname{Im}\left[\alpha_{m}\right] d}{2 m} \pm l \frac{\pi}{m}, \quad m=1, \ldots,(M-1)
$$

where $\pm l \pi / m$ is included because the oscillatory parts of the approximating exponentials in (3) are periodic with a period of $2 \pi$. That is, one needs to decide on the integer value $l$ for each $\alpha_{m}$ to bring the propagation constant into the irreducible Brillouin zone. Once the propagation constant $k_{z 1}$ in the equivalent homogenous medium is obtained, the effective permeability and permittivity values are calculated from the definitions of the Fresnel reflection coefficients (2a) and (2b) as

$$
\begin{aligned}
\left\{\begin{array}{ll}
\frac{\mu_{r 1}}{\mu_{r 0}} & \text { for TE waves } \\
\frac{\epsilon_{r 1}}{\epsilon_{r 0}} & \text { for TM waves }
\end{array}\right\}=\frac{\left(1+a_{0}\right) k_{z 1}}{\left(1-a_{0}\right) k_{z 0}} \\
\epsilon_{r 1} \mu_{r 1}=\left(\frac{c}{\omega}\right)^{2}\left(k_{0}^{2} \sin ^{2} \theta_{i}+k_{z 1}^{2}\right)
\end{aligned}
$$

where $a_{0}$ is the first term of the exponential approximation (3), $\theta_{i}$ is the angle of incidence, $c$ is the speed of light in vacuum, and $\omega$ is the radian frequency of operation. For normal incidence $\left(\theta_{i}=0^{\circ}\right)$, one of the two effective parameter sets in (6a) can be used because TE and TM waves differ only by a rotational symmetry and become degenerate.

\section{RESULTS AND DISCUSSIONS}

Since the subject matter of this study is to determine the effective constitutive parameters of finite extent periodic structures, two of the most popular periodic structures, namely, photonic crystals and metamaterials, were employed to demonstrate the proposed method. To facilitate clear and coherent discussions of the results relevant to these structures, the results of each structure are discussed and provided below. Moreover, it should be noted that the reflection coefficient data used throughout this study were obtained by numerical simulations, using Comsol Multiphysics by Comsol Inc. for 2-D photonic crystals, wires, and split-ring resonators (SRRs), and HFSS by Ansoft Inc. for metamaterials with 3-D metallic inclusions. As a final note on the implementation of the sampling strategy, one has to choose a biasing thickness $\left(N_{0} d\right)$ and the total number of samples $N$ judiciously for a given wavelength of operation. Since a detailed study of the effects of these choices to the effective parameters is rather lengthy and out of the scope of this paper, it would be sufficient to state that the approach is quite robust as long as the total number of samples cover a distance over which the main features of the generalized reflection coefficient can be included. Regarding the choice of the biasing thickness $N_{0} d$, it can easily be optimized by examining the quality of the effective parameters from the higher order terms in the exponential approximation once the reflection data is collected.

\section{A. Photonic Crystals}

Although photonic crystals are best described by the dispersion diagrams ( $\omega-k$ diagrams) when studying their bandgap behavior, their effective parameters can be of great use in the design of optical or microwave circuit elements in or on such structures, especially in their passbands. To the best of the authors' knowledge, there has not been much study on the determination of the effective parameters of such structures, except for an extensive study on the effective long-wavelength dielectric constants of heterogenous structures [13], [14], [24]-[26]. The concept of effective dielectric constant was introduced to estimate the equivalent dielectric constant of a composite structure in the quasi-static regime. In a recent study, effective refractive indices of 1-D photonic crystals, calculated from the phase factor of the transmission coefficient, were provided to study the influence of finite-size photonic crystals on the wave propagation [27]. In addition, in the study of photonic crystal fibers, the use of the effective refractive-index model for the cladding of a 2-D photonic crystal has made it possible to explain the large wavelength range for a single-mode operation of the photonic crystal fiber [28].

For the sake of validation, a simple 1-D photonic crystal with two alternating dielectric materials was employed with the following parameters: thickness and dielectric constant of the first layer are $160 \mathrm{~nm}$ and $\epsilon_{r 1}=1$, thickness and dielectric constant of the second layer are $40 \mathrm{~nm}$ and $\epsilon_{r 2}=13$, respectively. Although the structure is made up of alternating two different layers, the unit cell was defined symmetrically, as suggested in [20] for the well-defined boundaries of the equivalent slab; the length of the unit cell is $a=200 \mathrm{~nm}$, and the thicknesses and permittivities of the three layers involved in a unit cell are $(0.4 a, 1.0),(0.2 a, 13.0)$, and $(0.4 a, 1.0)$, respectively. Noting that the first bandgap of the photonic crystal starts at about the normalized frequency of $\omega a / c=1.32$ [29], the effective permittivity and permeability values were obtained up to the bandgap frequency for three different angles of incidence, as shown in Fig. 2. Before providing any discussion on the results, the field distributions in the periodic structure and in its homogeneous equivalent were obtained analytically for two different wavelengths of operation, and depicted in Fig. 3 for normal incidence and in Fig. 4 for $\theta_{i}=60^{\circ}$. The field distributions provide ultimate assessment of how good an equivalent homogeneous slab with the effective parameters approximates a periodic structure; however, they are rather difficult to obtain and are not practical for experimental study. As one of the strength of the method, the quality assessment can be performed by examining the higher order terms in the approximation of the generalized reflection coefficient (3), and because of that, the coefficients $a_{m}$ and exponents $\alpha_{m}$ of the approximations were provided in Table I.

First of all, it is observed throughout this study that the application of the method may result in positive imaginary values for 


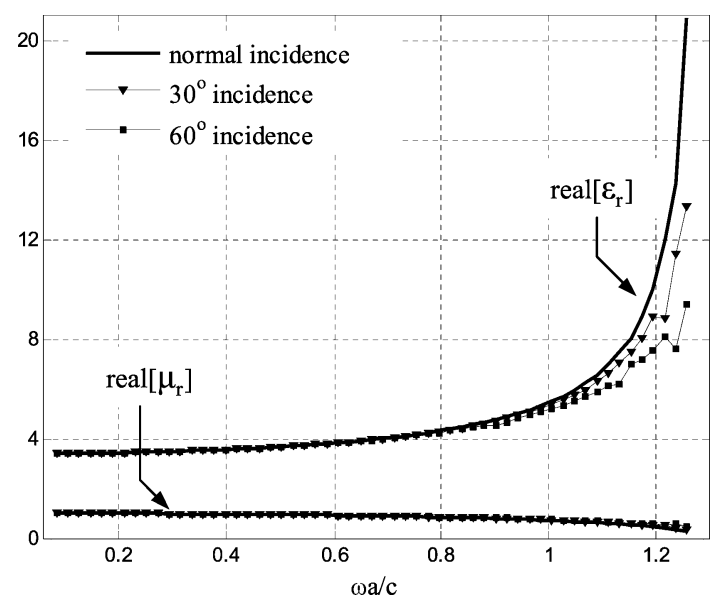

Fig. 2. Effective permittivity and permeability values of a 1-D photonic crystal for TE waves with $\theta_{i}=0^{\circ}, 30^{\circ}, 60^{\circ}$. Parameters of the unit cell: length $a=$ $200 \mathrm{~nm}$; thicknesses and permittivities of the three layers involved in a unit cell are $(0.4 a, 1.0),(0.2 a, 13.0)$, and $(0.4 a, 1.0)$, respectively.

the effective permittivity or permeability, which seems to be not consistent with the time dependence $e^{j \omega t}$ employed throughout this work and needs clarification. This behavior has nothing to do with any real physical mechanism, but it is just a result of mathematical operations. If the geometry under study is composed of lossless dielectric materials, as in the cases of photonic crystals, the resulting propagation constants are supposed to be purely real for the wavelengths of operation below the bandgap, i.e., the exponents of the generalized pencil-of-function approximation in (3) have to be dominantly imaginary, as observed from the data in Tables I and II. In addition, because of the fact that the term approximating the Fresnel reflection coefficient $a_{0}$ is a complex number in general, (6a) results in a complex $\epsilon_{r 1}$ or $\mu_{r 1}$. Consequently, the other effective parameter determined from (6b) has to compensate the imaginary part of the already determined parameter, resulting in opposite signs for the imaginary parts of these parameters, which are provided in the captions of the field plots for each case studied in this work. Attributing seemingly nonphysical behavior of the effective permeability (or permittivity) to the mathematical definitions of the effective parameters has increased the importance of the assessment of the validity of such parameters.

Assessment of the quality of the effective parameters can be performed either by using or processing information collected externally (noninvasive), or examining the field distributions in the original and equivalent structures (invasive), both of which are presented in this discussion. Of course, the noninvasive approach is easier to use and more practical, as it only requires data accessible externally. However, to see how well the homogeneous equivalent slab mimic the real periodic structure, the true test may be to compare the field distributions. Since the proposed method also provides the approximations of the higher order reflection terms at the front interface, as given in the tables, they can easily be compared to the multiple reflections from an equivalent homogenous slab, resulting in a good measure of the quality of the effective parameters. In Table I, the data was presented in the order as it was provided by the generalized pencil-of-function method, but the index $m$ had to be

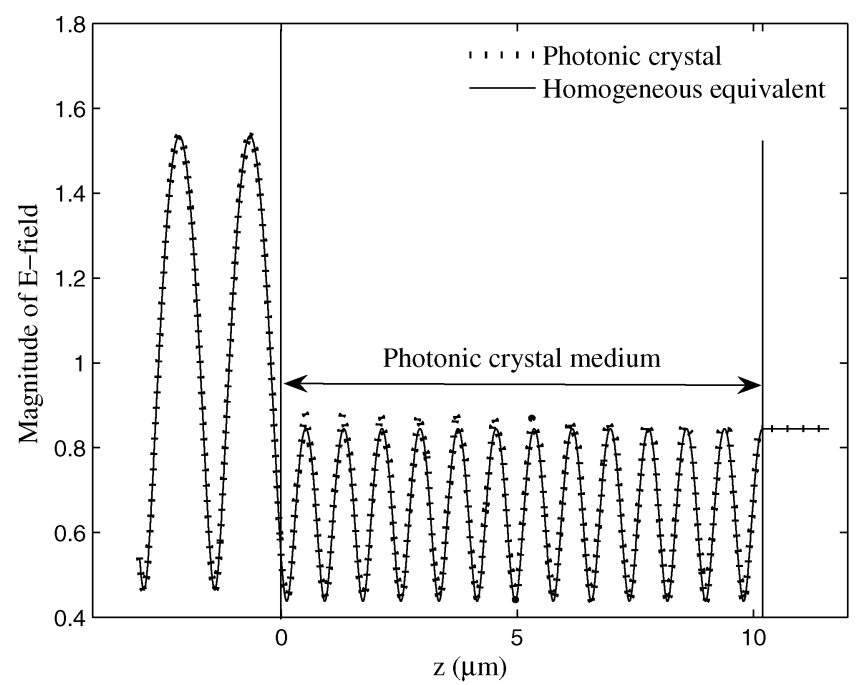

(a)

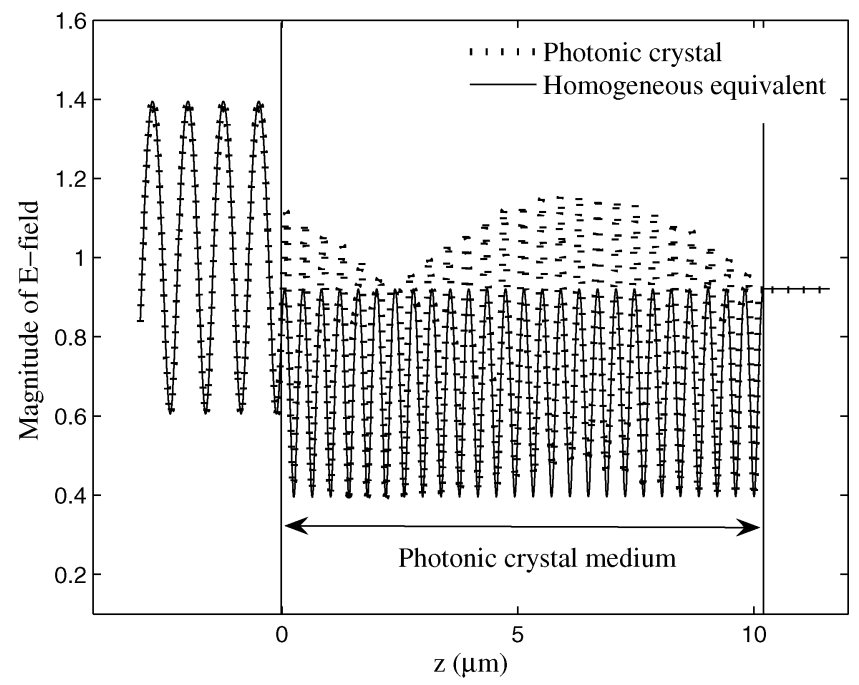

(b)

Fig. 3. Field distributions in the actual structure and its homogeneous equivalent slab under normal incidence at: (a) $\omega a / c=0.42\left(\epsilon_{\text {reff }}=3.59+\right.$ $\left.j 0.32 \times 10^{-5}, \mu_{\text {reff }}=0.96-j 0.11 \times 10^{-5}\right)$, and (b) $\omega a / c=0.84\left(\epsilon_{\text {reff }}=\right.$ $\left.4.46+j 0.0018, \mu_{\text {reff }}=0.83-j 0.0003\right)$. Parameters of a unit cell: length $a=200 \mathrm{~nm}$; thicknesses and permittivities of the three layers involved in a unit cell are $(0.4 a, 1.0),(0.2 a, 13.0)$, and $(0.4 a, 1.0)$, respectively.

decided based on the relative phase and magnitude values. That is, the term corresponding to Fresnel reflection coefficient must have a negligible phase, and the other terms are decided based on the decreasing magnitudes of the coefficients. For the best homogenous equivalent slab that mimics the periodic structure, all the exponents of the approximating terms must yield the same propagation constant $k_{z}(=k$ of the effective medium for the normal incidence) when the periodicity of each term is properly taken into account. Therefore, to assess the quality of the effective parameters, the normalized values of the wavenumber of the effective medium $(k a)$ were obtained with a proper choice of the integer $l$ for each term, and are provided in Table I. Resulting $k a$ values are observed to be almost equal with negligible differences (less than \pm 0.0001 ), implying that the periodic structure can be replaced by the homogeneous equivalent slab with the effective permittivity and permeability values obtained by 


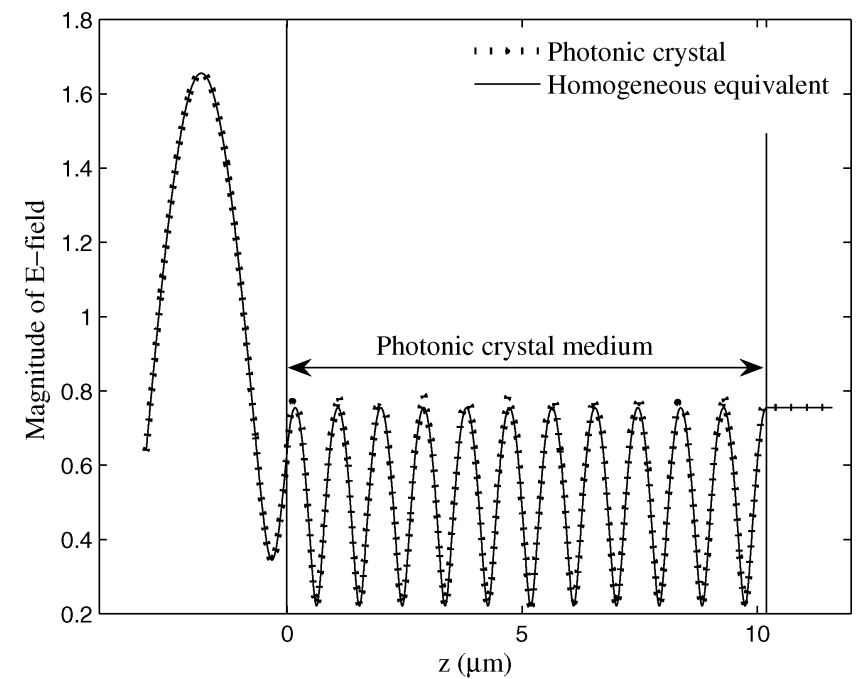

(a)

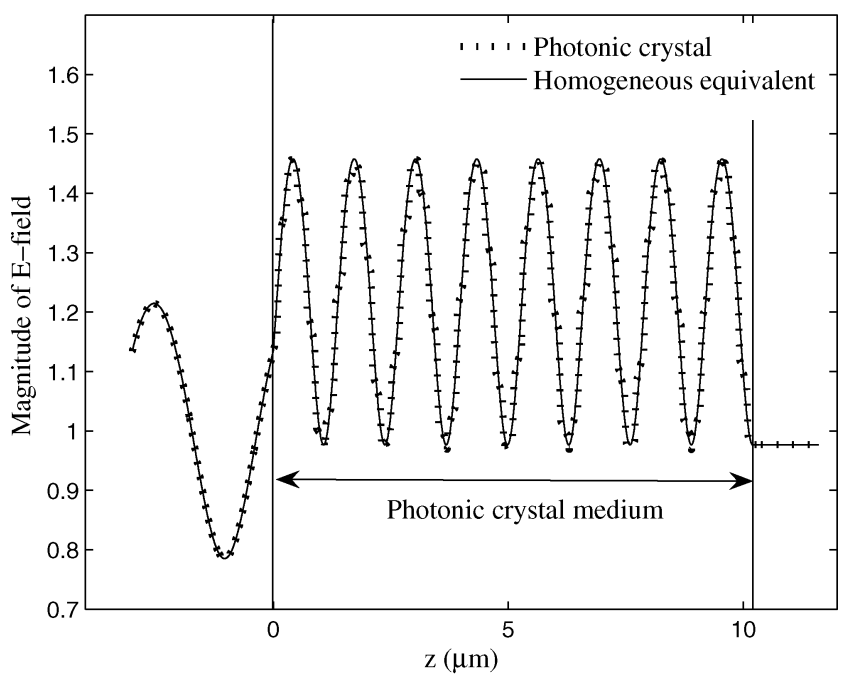

(b)

Fig. 4. Field distributions in the actual structure and in its homogeneous equivalent slab for $\omega a / c=0.42, \theta_{i}=60^{\circ}$. (a) TE polarization $\left(\epsilon_{\text {reff }}=3.58+\right.$ $\left.j 0.0014, \mu_{\text {reff }}=0.96-j 0.0004\right)$. (b) TM polarization $\left(\epsilon_{\text {reff }}=3.43+j 0.4 \times\right.$ $\left.10^{-7}, \mu_{\text {reff }}=0.60-j 0.7 \times 10^{-8}\right)$. Length $a=200 \mathrm{~nm}$, thicknesses and permittivities of the three layers involved in a unit cell are $(0.4 a, 1.0),(0.2 a, 13.0)$, and $(0.4 a, 1.0)$, respectively.

the proposed method. Note that the real parts of the exponents $\alpha_{m} a$ and the imaginary parts of the coefficients $a_{m}$ were negligibly small as compared to the corresponding imaginary parts and real parts, respectively, and were not included in Table I for the clarity of the presentation.

As stated above, the ultimate check for the quality of the effective parameters is to compare the field distribution along the actual structure to the one along its homogeneous equivalent. When this was done for the 1-D photonic crystal under the illumination of normal incident plane waves, at the same frequencies used in Table I, it was observed that the field distributions match quite well for the frequencies far from the gap region [see Fig. 3(a)]; however, they show some differences in the medium of the periodic structure for the frequencies closer to the bandgap frequency [see Fig. 3(b)]. Since the method is based on the reflection coefficient defined at the front interface, it is
TABLE I

PARAMETERS OF THE EXPONENTIAL APPROXIMATION FOR A 1-D PHOTONIC CRYSTAL

\begin{tabular}{|c|l|l|l|}
\hline \multicolumn{4}{|c}{$\omega a / c=0.42^{\dagger}$} \\
\hline$m$ & $a_{m}$ & $\alpha_{m} a$ & $k a$ \\
\hline 0 & -0.317 & $j 0.0$ & \\
\hline 4 & $2.89 \times 10^{-4}$ & $j 0.0519$ & 0.7789 \\
\hline 1 & 0.285 & $-j 1.5578$ & 0.7789 \\
\hline 2 & $2.86 \times 10^{-2}$ & $-j 3.1156$ & 0.7789 \\
\hline 3 & $2.88 \times 10^{-3}$ & $j 1.6098$ & 0.7789 \\
\hline
\end{tabular}

\begin{tabular}{|c|l|l|l|}
\hline \multicolumn{4}{|c}{$\omega a / c=0.84$} \\
\hline$m$ & $a_{m}$ & $\alpha_{m} a$ & $k a$ \\
\hline 2 & -0.398 & $j 0.0$ & \\
\hline 4 & $5.3 \times 10^{-2}$ & $-j 0.1601$ & 1.6108 \\
\hline 1 & 0.335 & $j 3.0615$ & 1.6108 \\
\hline 3 & $8.42 \times 10^{-3}$ & $-j 0.3195$ & 1.6107 \\
\hline
\end{tabular}

$\dagger a(=200 \mathrm{~nm})$ is the length of a unit-cell

TABLE II

PARAMETERS OF THE EXPONENTIAL APPROXIMATION FOR A 2-D PHOTONIC CRYSTAL

\begin{tabular}{|c|l|l|l|}
\multicolumn{4}{|c|}{$\omega a / c=0.86^{\dagger}$} \\
\hline$m$ & $a_{m}$ & $\alpha_{m} a$ & $k a$ \\
\hline 0 & $-0.21+j 9.0 \times 10^{-4}$ & $-j 0.0$ & \\
\hline 2 & $2.2 \times 10^{-3}+j 8.9 \times 10^{-3}$ & $+j 1.33$ & 1.23747 \\
\hline 1 & $-0.16-j 0.125$ & $-j 2.47$ & 1.23746 \\
\hline 3 & $2.0 \times 10^{-4}-j 4.0 \times 10^{-4}$ & $-j 1.14$ & 1.23745 \\
\hline 4 & $<0.5 \times 10^{-4}-j 0.5 \times 10^{-4}$ & $+j 2.67$ & 1.23753 \\
\hline
\end{tabular}

$\dagger a(=400 \mathrm{~nm})$ is the length of a unit-cell

expected that the fields in this region are primarily enforced, especially when the higher order terms in the approximation provide close effective propagation constants. For the validation of the method under arbitrary angles of incidence, the method was applied to the same 1-D photonic crystal for various angles of incidence with TE and TM polarizations, and the field distributions corresponding to the angle of incidence of $\theta_{i}=60^{\circ}$ were provided for TE and TM polarizations in Fig. 4, as compared to the exact field distributions in the photonic crystal.

Once the validation of the method has been performed on a simple 1-D photonic crystal, for which the reflection data and the field distributions were calculated analytically, a more complex geometry, i.e., a 2-D photonic crystal, as shown in the inset of Fig. 5, was considered next. The structure is a square array of dielectric columns with radius $r=0.2 a$ and dielectric constant $\epsilon_{r}=8.9$ embedded in air, and has the bandgap starting around at $\omega a / c=1.7$ [29]. The reflection data needed to implement the method, and the field distributions needed to compare the field distributions along its homogeneous equivalent were obtained by using Comsol Multiphysics by Comsol Inc. Using the reflection coefficient data due to a normal incident plane wave, the effective parameters were calculated by using the proposed method with five exponentials. To assess the performance of the method for this more complicated structure, whose analysis cannot be performed analytically, the effective propagation constant of the structure corresponding to each term 


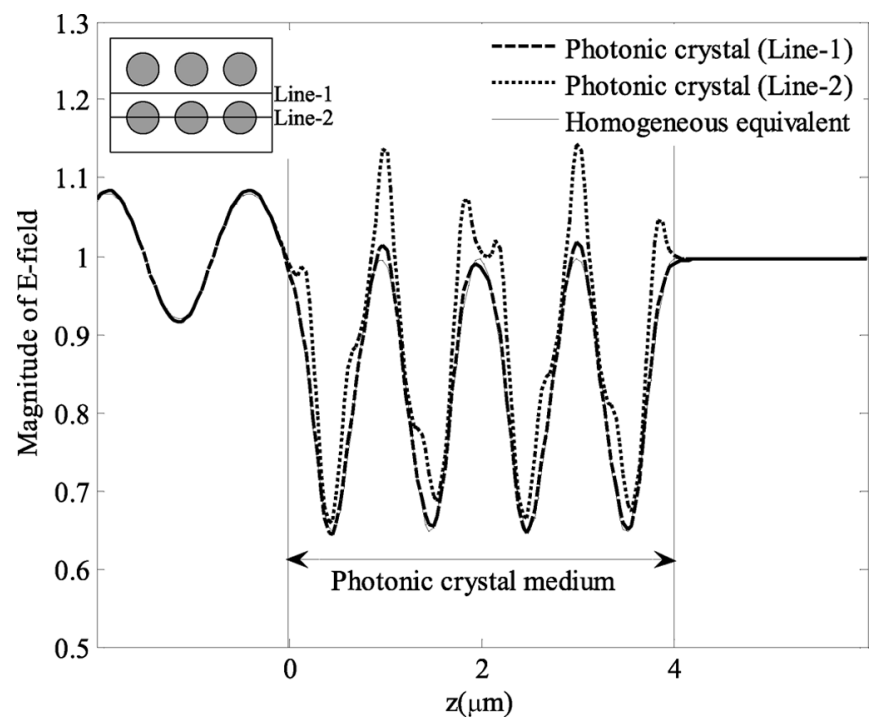

Fig. 5. Field distributions in the actual structure and in its homogenous equivalent slab with the effective parameters of $\epsilon_{\text {reff }}=2.22-j 0.004$ and $\mu_{\text {reff }}=$ $0.94+j 0.002$ at $\omega a / c=0.86$. Parameters of a unit cell: length $a=400 \mathrm{~nm}$; dielectric columns with radius $r=0.2 a$, and dielectric constant $\epsilon_{r}=8.9 \mathrm{em}$ bedded in air.

in the exponential approximation was provided in Table II. Except for the Fresnel reflection term, the remaining four terms have resulted in almost the same effective propagation constant, indicating that the homogeneous equivalent slab with the determined effective parameters mimics the structure almost perfectly. To further verify this conclusion, the field distributions along two different cuts of the periodic structure, one passing over the background material (Line-1) and the other over the dielectric columns (Line-2), as shown in the inset of Fig. 5, were compared to the field distribution obtained from its homogeneous equivalent slab, Fig. 5. As expected from the study of the effective propagation constants corresponding to the higher order terms, the field distributions match almost perfectly in the medium of incidence, no matter which cut is chosen. Moreover, they match quite well along Line-1, while there is expected deviation along Line- 2 in the region of the periodic structure. Although no explicit information on the transmitted wave was used in the application of the method, the fields in the exit medium obtained from the equivalent slab configuration match quite well to those obtained from the actual structure.

In conclusion, the proposed method works quite well in determining the effective constitutive parameters of photonic crystals, not only for normal incident plane waves, but also for a plane wave of any polarization and any angles of incidence. Moreover, the higher order terms of the exponential approximation, which are not directly used in determining the effective parameters, offer a good measure of how good the homogeneous equivalent slab can substitute the actual structure.

\section{B. Metamaterials With Metallic Inclusions}

Metamaterials, which are also referred to as left-hand materials, negative index materials, and double-negative materials in the literature, are also popular periodic structures and are investigated in this section with a view to determine the effective parameters of their homogeneous equivalent slabs. Contrary to

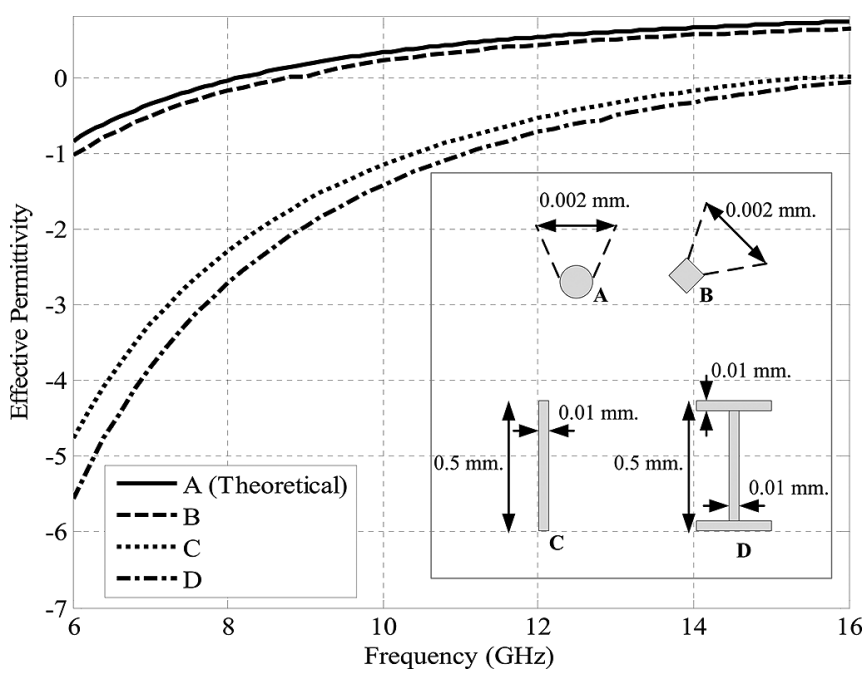

Fig. 6. Real parts of the effective permittivities of periodic structures composed of infinite long wires with circular (A), square (B), rectangular (C), and rotated $H$ (D) cross sections, as shown in the inset.

the photonic crystals, which are mainly characterized by the dispersion diagrams, metamaterials are usually described by their equivalent material parameters, either retrieved from the reflection and/or transmission data [18]-[21] or determined by averaging the local fields [15]-[17]. It should be stressed here that the method proposed in this paper is different from the retrieval procedures, even though both are based on the reflection and/or transmission coefficients, and obviously different from the averaging approaches, which have been reviewed in details recently in [17].

The proposed method was first applied to the simple 2-D constituting components of metamaterials, namely, wires and SRRs, for which effective permittivity and permeability values may be known analytically. It should be noted that, during the study of metamaterials, reflection data starting from one unit cell up to maximum of 20 unit cells were collected for a frequency band and used to determine the effective parameters of the structures. As a first example, periodic wire structures with four different cross sections, designated by A-D in the inset of Fig. 6, were studied for a normal incident plane wave. The motivation for this example was that a medium composed of periodic wires with circular cross sections, structure A, can be characterized by a well-defined expression of its permittivity value as a function of frequency [30]

$$
\begin{aligned}
\epsilon_{\mathrm{reff}} & =1-\frac{\omega_{p}^{2}}{\omega\left(\omega-\frac{j \epsilon_{\circ} u^{2} \omega_{p}^{2}}{\pi r^{2} \sigma}\right)} \\
\omega_{p}^{2} & =\frac{2 \pi c^{2}}{u^{2} \ln \left(\frac{u}{r}\right)}
\end{aligned}
$$

where $\omega_{p}$ is the plasma frequency, $u\left(=5 \times 10^{-3} \mathrm{~m}\right)$ is the periodicity of the wire structure, and $r\left(=10^{-6} \mathrm{~m}\right)$ and $\sigma(=$ $6 \times 10^{7} \mathrm{~S} / \mathrm{m}$ ) are the radius and the conductivity of the wire, respectively. Hence, the permittivity variations of wires with different cross sections can be studied using the proposed method, and compared to those obtained by the analytical expression of 
TABLE III

PARAMETERS OF EXPONENTIAL APPROXIMATION FOR CONFIGURATION B IN FIG. 6

\begin{tabular}{|c|l|l|l|}
\multicolumn{5}{c}{$f=10 \mathrm{GHz}$, length of a unit-cell $u=0.5 \mathrm{~cm}$} \\
\hline$m$ & $a_{m}$ & $\alpha_{m} u$ & $k u$ \\
\hline 4 & $<0.5 \times 10^{-4}(1+j)$ & $1.26+j 2.39$ & 0.487 \\
\hline 0 & $0.39+j 0.032$ & $2.0^{-4}+j 6.0 \times 10^{-4}$ & \\
\hline 1 & $-0.18+j 0.25$ & $-0.096-j 0.99$ & 0.495 \\
\hline 2 & $0.013+j 0.044$ & $-0.20-j 1.94$ & 0.485 \\
\hline 3 & $3.1 \times 10^{-3}+j 0.0$ & $-0.077-j 2.87$ & 0.478 \\
\hline
\end{tabular}

a periodic wire structure with circular cross section [see (7)]. As expected, the effective permittivity variation of the structure with square cross section exhibits almost the same trend with very close values as that of circular cross section obtained by (7). However, the other wire configurations show stronger variations with frequency, and exhibit more negative permittivity values over the same frequency band, as shown in Fig. 6. It should also be noted that since structures $\mathrm{C}$ and $\mathrm{D}$ are geometrically anisotropic, they are likely to result in anisotropic behavior in their effective permittivity values.

For the assessment of the effective parameters, the values of the propagation constant corresponding to the terms in the exponential approximation were provided at $10.0 \mathrm{GHz}$ in Table III, for configuration B in Fig. 6. Although the effective propagation constants are very close, some deviation becomes visible for the terms whose coefficients are relatively small. To complete the assessment, the field distribution in the equivalent homogenous medium, whose effective parameters are $\epsilon_{\text {reff }}=0.22-j 0.04$ and $\mu_{\text {reff }}=1.09-j 0.01$ at $10.0 \mathrm{GHz}$, was compared to that in the actual periodic structure, as shown in Fig. 7. For the actual periodic structure, a medium composed of eight stacks of configuration $\mathrm{B}$, with the total width of $4.0 \mathrm{~cm}$, in a free-space environment was analyzed by using Comsol Multiphysics by Comsol Inc. It is observed that the field distribution (magnitude of $E$-field) in the equivalent homogeneous structure follows, almost exactly, the one in the actual structure (Fig. 7). Therefore, looking at the data given in Table III and the curves in Fig. 7, it is safe to state that the equivalent homogeneous slab with the effective parameters can successfully replace the periodic structure at $10.0 \mathrm{GHz}$ under normal incidence.

For the sake of completeness, a medium with periodic SRRs, whose geometry and dimensions were given in the inset and caption of Fig. 8, was characterized by its effective parameters using the proposed method. The SRRs employed in this study were assumed to be made of copper, to extend to infinity in the perpendicular direction to the plane of periodicity, and to be surrounded by vacuum. After having collected the reflection data due to a normal incident plane wave over a frequency band, the effective permeability of the structure was obtained by using the proposed method, as shown in Fig. 8. It should be noted that, in the case of periodic wires, the propagation constant and field distributions were obtained at $10.0 \mathrm{GHz}$ at which the effective permittivity value is positive. This was an intentional choice as a medium with negative permittivity would have only supported evanescent waves, and consequently, there would be no multiple reflections. To eliminate any concern on the applicability of the proposed method to media with one of the effective parameters

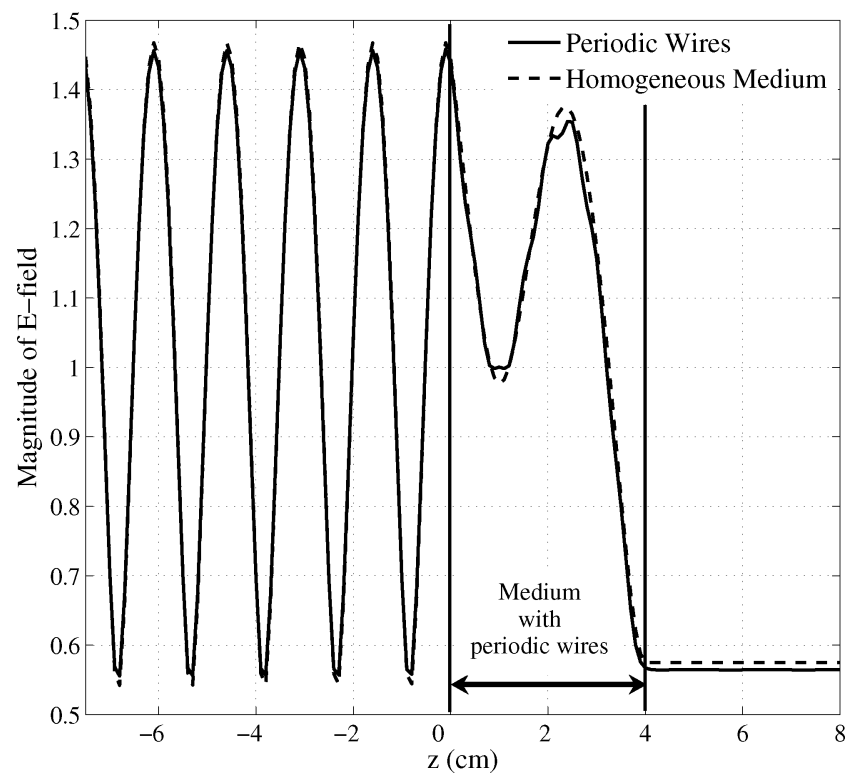

Fig. 7. Magnitudes of $E$-field in the periodic medium and in its homogeneous equivalent slab at $10.0 \mathrm{GHz}$. The effective parameters are $\epsilon_{\text {eff }}=0.22-j 0.04$ and $\mu_{\mathrm{eff}}=1.09-j 0.01$.

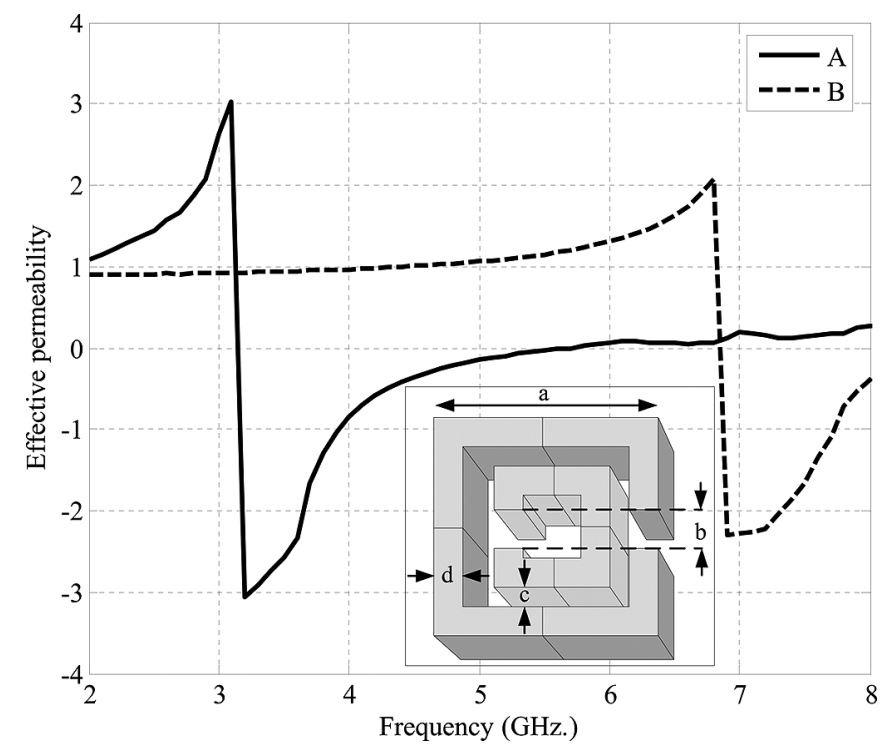

Fig. 8. Real parts of effective permeability corresponding to periodic structures of two different SRRs with the unit-cell size of $5 \mathrm{~mm}$. SRR A: $a=4.4 \mathrm{~mm}$, $b=0.6 \mathrm{~mm}, c=0.3 \mathrm{~mm}, d=0.2 \mathrm{~mm}$; SRR B: $a=3.0 \mathrm{~mm}, b=0.5 \mathrm{~mm}$, $c=0.3 \mathrm{~mm}$, and $d=0.2 \mathrm{~mm}$.

less than zero, the magnetic field distribution for SRR A with the effective parameters at $4.0 \mathrm{GHz}\left(\epsilon_{\text {eff }}=7.12+j 0.13\right.$ and $\left.\mu_{\mathrm{eff}}=-0.86-j 0.02\right)$ was provided and compared to the exact field distribution along the actual structure in Fig. 9. To complete this part of the study, the propagation constants and the magnetic field distributions were also provided in Table IV and in Fig. 10, respectively, for SRR B at $5.0 \mathrm{GHz}$, at which the effective parameters are positive quantities as $\mu_{\text {reff }}=1.06+j 0.00$, $\epsilon_{\mathrm{reff}}=2.26-j 0.01$. Note that the results provided for this example in Table IV and Fig. 10 were obtained by using five exponentials, just to be consistent with the other examples provided earlier. However, different number of exponentials were used in 


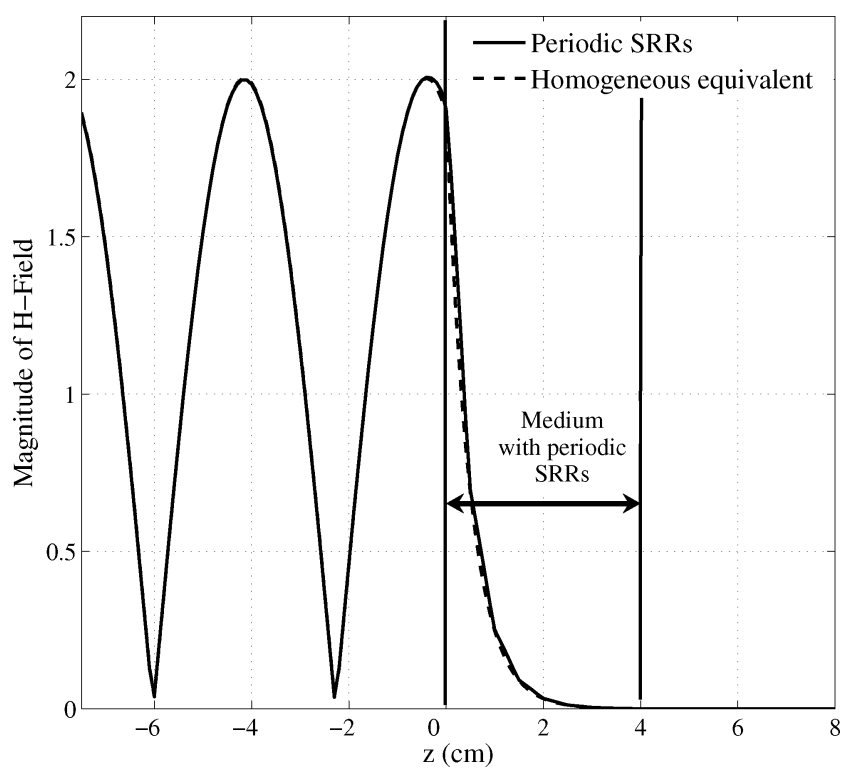

Fig. 9. Magnitudes of $H$-field in SRR A in Fig. 8 and in its homogenous equivalent slab at $4.0 \mathrm{GHz}$. The effective parameters are $\mu_{\mathrm{eff}}=-0.86-j 0.02$ and $\epsilon_{\text {eff }}=7.12+j 0.13$.

TABLE IV

PARAMETERS OF THE EXPONENTIAL APPROXIMATION FOR SRR-B IN FIG. 8

\begin{tabular}{|c|l|l|l|}
\hline \multicolumn{5}{|c}{$f=5.0 \mathrm{GHz}$, length of a unit-cell $u=0.5 \mathrm{~cm}$} \\
\hline$m$ & $a_{m}$ & $\alpha_{m} u$ & $k u$ \\
\hline 1 & $0.03+j 0.18$ & $-j 1.6213$ & 0.81065 \\
\hline 2 & $6.0 \times 10^{-3}-j 2.0 \times 10^{-3}$ & $-9.0^{-4}+j 3.04$ & 0.81035 \\
\hline 0 & $0.187-j 1.6 \times 10^{-3}$ & $-(2.0+j 2.0) \times 10^{-4}$ & \\
\hline 3 & $1.0 \times 10^{-4}-j 1.0 \times 10^{-3}$ & $-0.20+j 0.26$ & 1.0039 \\
\hline 4 & $-j 3.0 \times 10^{-4}$ & $-0.075+j 1.37$ & 0.6143 \\
\hline
\end{tabular}

(3) to approximate the reflection data, and the corresponding $k$ values for each term in the approximation as well as the field distributions were obtained and studied carefully. As a result, a few remarks are considered to be worth noting, which are: 1) no matter how many terms were used in the approximation of the reflection data, the exponents of the terms beyond the third term were not consistent with the expected multiple reflections, either due to small coefficients of these terms or to scattering from the metallic inclusions that cannot be accounted for by an homogenous equivalent slab and 2) although the field distributions along the actual structures were predicted quite well by the equivalent homogeneous slabs in the incident and transmitted layers (Layer-0 and Layer-2, respectively), the difference in the periodic region was more pronounced as compared to the structures studied earlier, even though the fields were sampled along a cut between the two SRRs.

After having applied the proposed method to the 2-D constituting metallic inclusions of metamaterials separately, it is now time to study the method for the medium composed of periodic SRRs and wires together in a 3-D setting. For the study of such materials, being of infinite extent in transverse directions, namely, $x$ and $y$, and of the finite extent in the direction of propagation $z$, a normal incident plane wave with $E_{x}$ and $H_{y}$ components was used to implement the method proposed in this

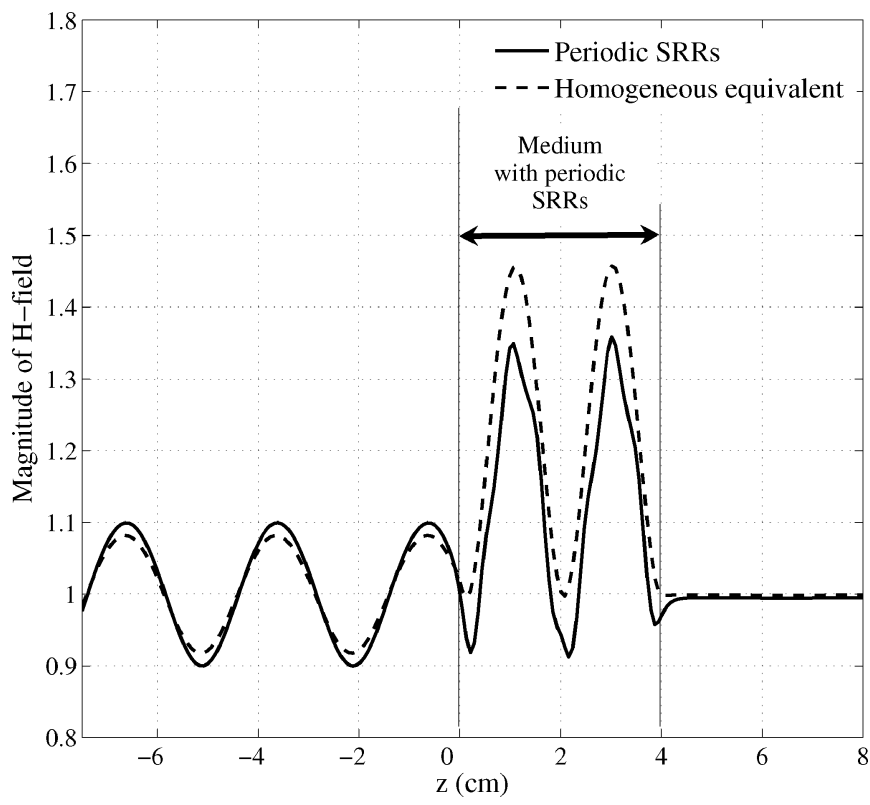

Fig. 10. Magnitudes of $H$-field in SRR B and in its homogenous equivalent $\mathrm{slab}$ at $5.0 \mathrm{GHz}$. The effective parameters are $\mu_{\text {reff }}=1.06+j 0.00$ and $\epsilon_{\text {reff }}=$ $2.26-j 0.01$.

paper. The details of the geometry are the same as those studied in [21], except that split-ring and wire structures are assumed to be perfect conductors with zero thicknesses to reduce the required memory and computation cost: a cubic unit cell with a dimension of $u=2.5 \mathrm{~mm}$, dipole and SRR are printed on the opposite sides of a 0.25 -mm-thick substrate with $\epsilon_{r}=4.4$ and loss tangent of 0.02 , printed dipole with the width of $0.14 \mathrm{~mm}$ runs through the length of the unit cell, and dimensions of the SRR, as referenced to the inset of Fig. 8 , are $a=2.2 \mathrm{~mm}$, $b=0.3 \mathrm{~mm}, c=0.15 \mathrm{~mm}$, and $d=0.2 \mathrm{~mm}$. In addition, throughout the study of these 3-D metamaterials, calculation of the reflection coefficient at the front interface was performed by HFSS by Ansoft Inc., with the use of an efficient implementation suggested in [31]. For this study, a good number of reflection coefficient data were collected, starting from one unit cell up to 20 unit cells in the direction of propagation, over a frequency band of 5.0-15.0 GHz.

Since the study of the metamaterial was performed over a frequency band, the proposed method was implemented for each frequency using 20 samples of the generalized reflection coefficient at the front interface. Before providing any result, it should be noted that metamaterial structures exhibit negative effective values for either permittivity or permeability, but not both, over some frequency bands, where the wave is dominantly attenuating in the structure, resulting in negligible higher order reflection terms at the front interface. For such cases, the exponential approximation results in two dominant exponentials; one corresponds to Fresnel's reflection and the other provides a propagation constant with a large negative imaginary part, revealing the evanescent wave nature in the structure. However, over the bands where the structure behaves like double-negative or double-positive material, the higher order terms of the generalized reflection coefficient become significant. To give an idea how the exponential approximations look like for the metamaterial operating at three different frequencies corresponding to 
TABLE V

PARAMETERS OF THE EXPONENTIAL APPROXIMATION FOR THE METAMATERIAL

$f=5.0 \mathrm{GHz}$
\begin{tabular}{|c|l|l|l|}
\hline$m$ & $a_{m}$ & $\alpha_{m} u$ & $k u$ \\
\hline 0 & $-0.90+j 0.43$ & $\approx 0.0+j 0.0$ & \\
\hline 1 & $-1.10+j 1.87$ & $-3.73-j 1.46$ & 0.7294 \\
\hline
\end{tabular}

$f=10.8 \mathrm{GHz}$

\begin{tabular}{|c|l|l|l|}
\hline$m$ & $a_{m}$ & $\alpha_{m} u$ & $k u$ \\
\hline 0 & $-0.35-j 0.09$ & $\approx 0.0+j 0.0$ & \\
\hline 1 & $0.34+j 0.04$ & $-0.11+j 0.78$ & -0.389 \\
\hline 2 & $3.5 \times 10^{-3}-j 0.1$ & $-0.33+j 1.69$ & -0.423 \\
\hline 3 & $2.4 \times 10^{-2}-j 1.3 \times 10^{-3}$ & $-0.22-j 1.59$ & -0.782 \\
\hline 4 & $-1.4 \times 10^{-2}+j 1.1 \times 10^{-3}$ & $-0.1-j 2.63$ & -0.456 \\
\hline
\end{tabular}

\begin{tabular}{|c|l|l|l|}
\hline \multicolumn{4}{|c}{$f=15.0 \mathrm{GHz}$} \\
\hline$m$ & $a_{m}$ & $\alpha_{m} u$ & $k u$ \\
\hline 0 & $-0.27+j 9.8 \times 10^{-3}$ & $\approx 0.0+j 0.0$ & \\
\hline 1 & $0.25-j 0.015$ & $-0.041-j 1.35$ & 0.673 \\
\hline 2 & $0.029-j 1.9 \times 10^{-4}$ & $-0.148-j 2.72$ & 0.679 \\
\hline 3 & $4.3 \times 10^{-3}-j 5.4 \times 10^{-3}$ & $-0.206+j 2.27$ & 0.669 \\
\hline 4 & $-1.1 \times 10^{-3}+j 1.8 \times 10^{-3}$ & $-0.025-j 2.09$ & 1.047 \\
\hline 5 & $-6.5 \times 10^{-4}-j 1.7 \times 10^{-3}$ & $-0.053+j 1.28$ & 0.500 \\
\hline
\end{tabular}

these three bands, the coefficients, exponents, and the effective propagation constants were given in Table V. Since the effective propagation constants resulting from the higher order exponential terms were used to measure the quality of the homogeneous equivalent slab in the previous examples, they were also examined for the metamaterial: for both double-negative $(f=10.8 \mathrm{GHz})$ and double-positive $(f=15.0 \mathrm{GHz})$ cases, $k u$ values for the dominant terms are sufficiently close, implying that the equivalent homogeneous slabs can successfully imitate the metamaterial, though not as good as the cases studied for the photonic crystals. This may partly be attributed to the strong scattering nature of the metallic inclusions. To better assess the quality of homogenization, the field distributions in the actual structure and in its homogeneous equivalent were also obtained and examined at the same frequencies as the ones used in Table V, as shown in Fig. 11, except at $5.0 \mathrm{GHz}$ for the obvious reason of a trivial behavior of the field distribution (see Fig. 9 for the split-ring structure). Since the field distribution in the periodic region is dependent on the path along which the field is sampled, the magnitudes of the electric fields were shown in Fig. 11 along two different paths in the direction of propagation; one passing through the center of a unit cell (between the SRR and dipole) and the other along the edge of a unit cell (between two unit cells). Since the former path (center) passes very close by the conducting split-rings and dipole structures, the electric field strength along this path was observed to be quite small, as expected. However, along the latter path (edge), being away from any geometrical discontinuities and conducting objects, the electric field distribution was expected and observed to be closer to that obtained from the homogenous equivalent (see Fig. 11). In addition, the field strengths outside the periodic medium have shown very good agreement no matter which path was chosen.

Although the method was applied to the metamaterial over a large frequency band $(5.0-15.0 \mathrm{GHz})$, the verification of the re-

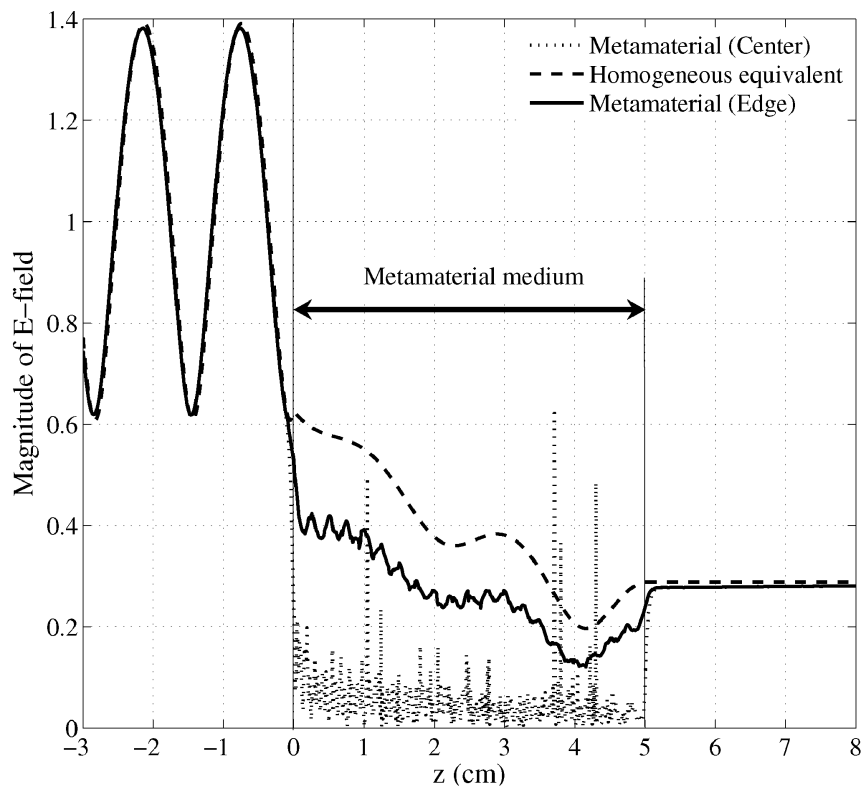

(a)

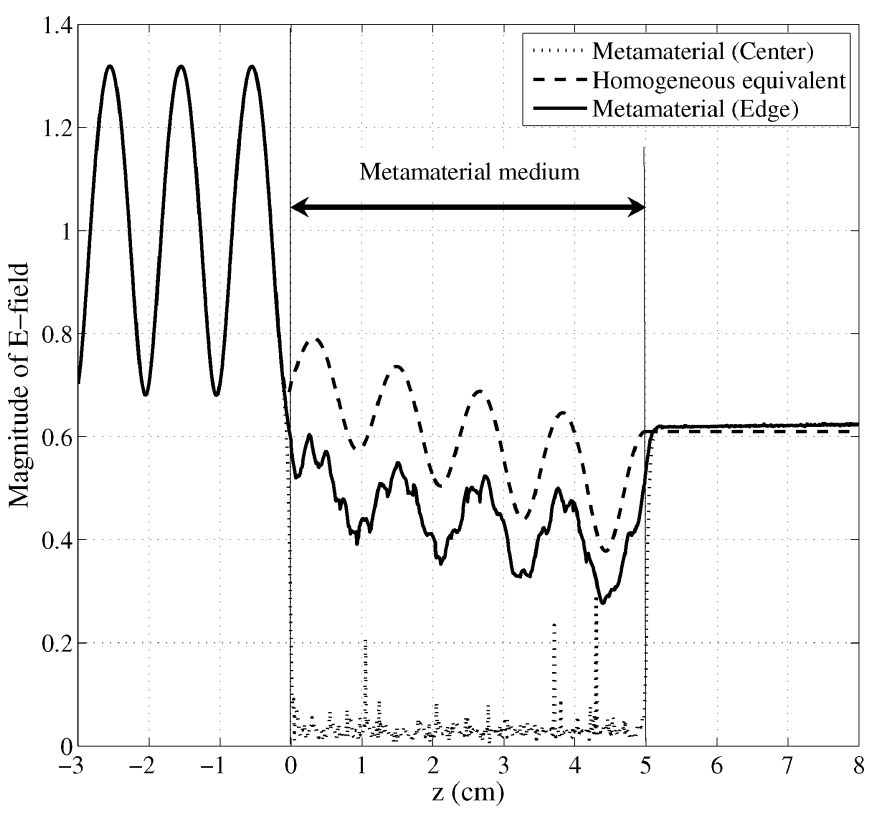

(b)

Fig. 11. Magnitudes of $E$-field in the metamaterial and in its homogeneous equivalent slab at: (a) $10.8 \mathrm{GHz}$ (both parameters are negative) and (b) $15.0 \mathrm{GHz}$ (both parameters are positive).

sults, and in turn, of the method, has been performed only over some discrete representative frequencies by means of propagation constants and field distributions. Since it would be more informative to see the performance of the method as applied to the metamaterial over the entire band, the effective permittivity and permeability values were obtained first, as shown in Fig. 12. The scattering parameter $S_{11}$, calculated directly from the HFSS data, for the actual structure with 20 unit cells in the direction of propagation are then compared to that obtained analytically from its homogeneous equivalent (using the data provided in Fig. 12), as depicted in Fig. 13. It is observed that the agreement of the scattering parameters are extremely good over the 


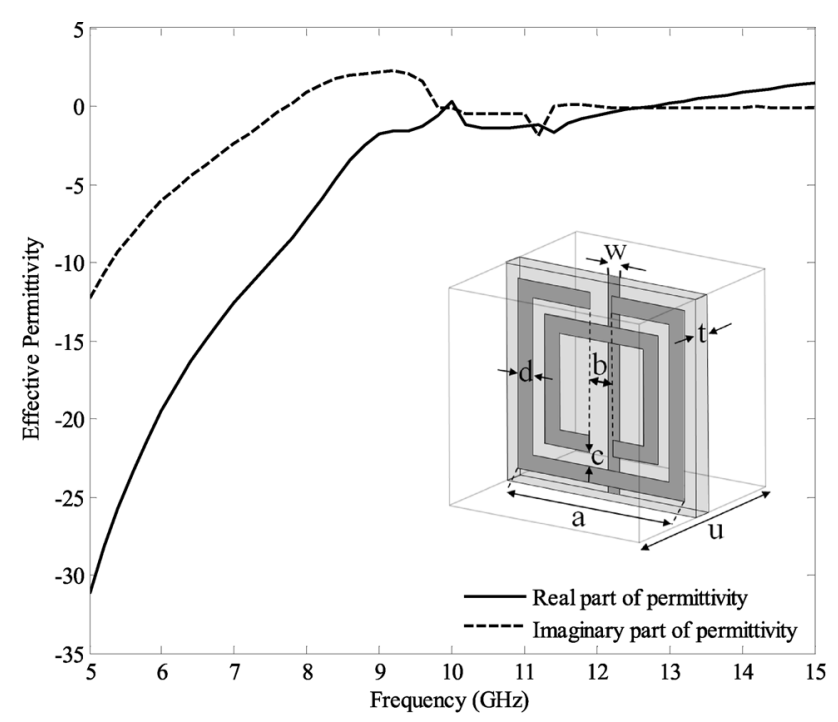

(a)

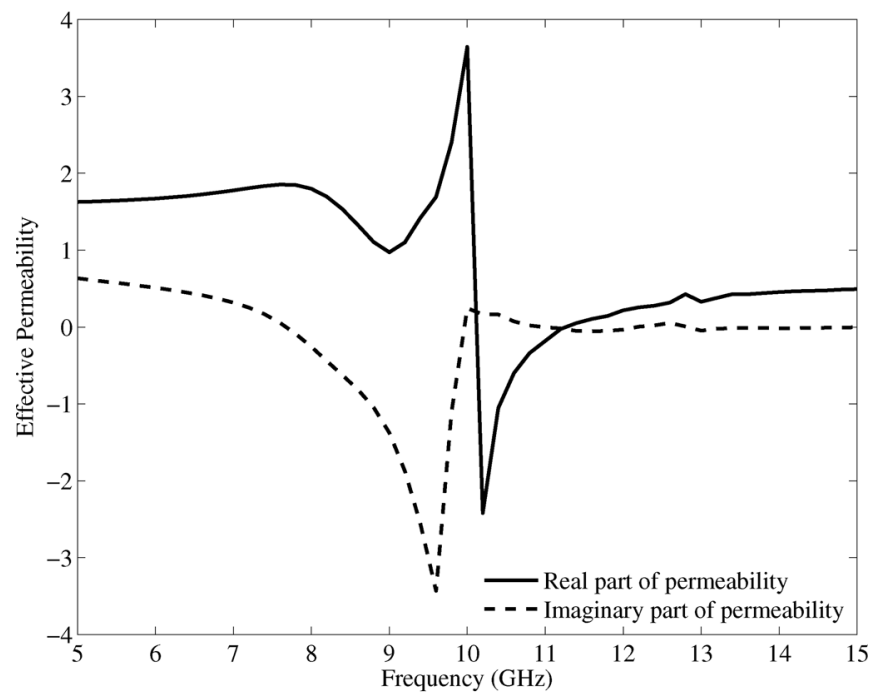

(b)

Fig. 12. Effective parameters of the metamaterial over a frequency band. (a) $\epsilon_{\text {reff }}$. (b) $\mu_{\text {reff }}$.

entire band, and that the frequency behavior of the effective parameters are consistent with the expectations. In addition, the plots of the effective parameters over the frequency band provide good comparisons to the same curves for a single unit cell of the same structure in [21], and demonstrate how the effective parameters of the periodic structure with arbitrary length would be different from the retrieved parameters of a fixed length of the structure.

After having studied so many cases, it would be instructive to provide a few comments on the method with a view to improve it further. Starting with its strengths, it should be emphasized that the method is capable of providing the effective parameters for any arbitrary angle of incidence, capable of resulting in by-products that would help measure the quality of the equivalent homogeneous slab easily, capable of handling any structure including nonperiodic structures and periodic structures with defects provided the reflection data can be obtained by some means. However, there are some weaknesses of the method, as

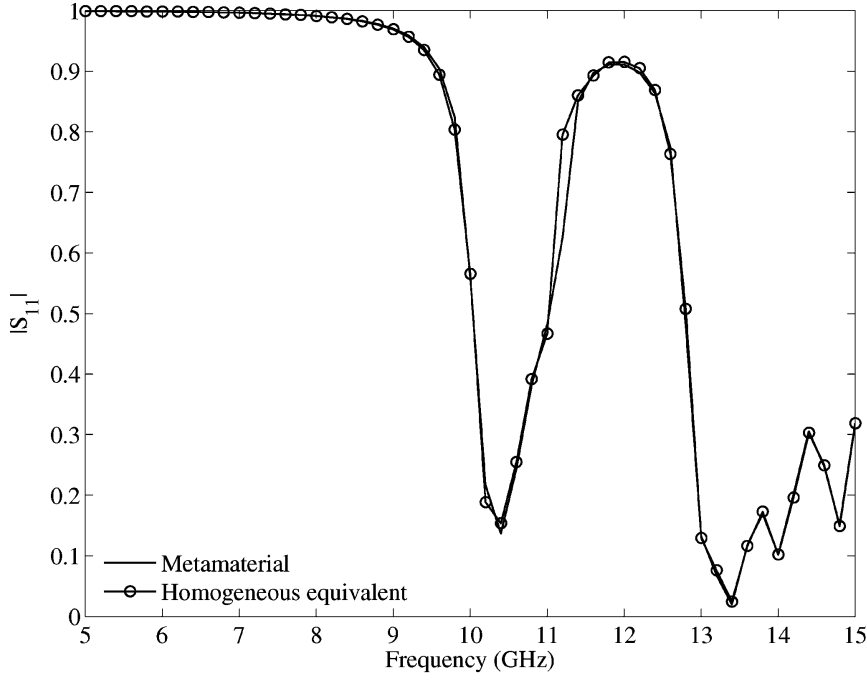

(a)

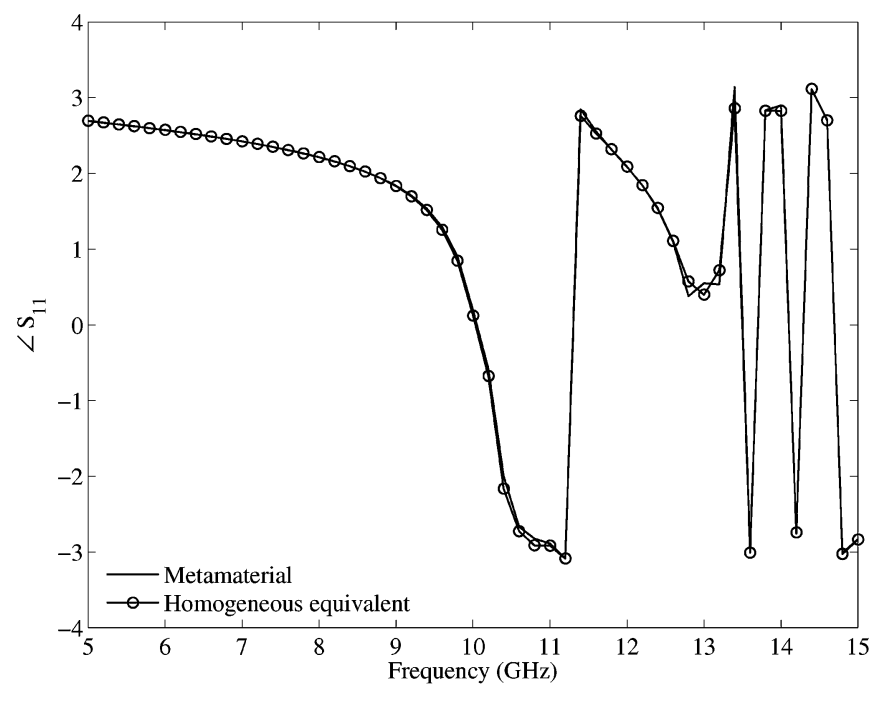

(b)

Fig. 13. Comparison of $S_{11}$ over a frequency band obtained from the homogeneous equivalent slab and from the actual metamaterial. (a) Magnitude. (b) Phase.

it was presented, which are listed as follows with their possible remedies.

1) The method only uses the reflection data as a function of the thickness of the structure, which may create problems in cases of small higher order reflections from the structure composed of lossy materials or single-negative materials. The remedy for this is simple, and perhaps it should be implemented for all cases, which is to use the transmission data together with the reflection data to extract the propagation constant with better accuracy.

2) Since the collection of the reflection and/or transmission data from a complex structure usually requires extremely high computation power, it may not be feasible to study some nonperiodic structures or periodic structures with defects. There is no obvious and immediate remedy for this, except to develop a fast accurate EM analysis tool for the specific problem at hand. 
3) The implementation of the method concerning the choices of the number of initial layers, number of samples of the reflection data, and number of exponentials are to be studied in details, as these parameters are most likely to be influenced by the effective refractive index of the structure. Considering the fact that the smaller the effective refractive index of the structure, the larger the effective wavelength in the medium, the number of samples need to be increased in order to capture the relevant features of the reflection coefficient.

\section{CONCLUSION}

In this study, a simple method was introduced to find an equivalent homogeneous slab that could replace a periodic structure of an arbitrary length when illuminated by a plane wave with a given angle of incidence and polarization. The method determines the effective wavenumber and effective electric and magnetic parameters of the equivalent homogeneous slab from a set of reflection data collected for different thicknesses of the periodic structure. As one of the important features of the method, the effective parameters obtained are not dependent on the length of the periodic structure, and therefore, once obtained they can be used for the same structure with different lengths. The method was successfully demonstrated on 1-D and 2-D photonic crystals, and metamaterials with 2-D and 3-D metallic inclusions.

\section{REFERENCES}

[1] S. G. Johnson and J. D. Joannopoulos, Photonic Crystals: The Road from Theory to Practice. Boston, MA: Kluwer, 2002.

[2] IEEE Trans. Antennas Propag. (Special Issue), vol. 51, no. 10, Oct. 2003.

[3] IEEE Trans. Antennas Propag. (Special Issue), vol. 53, no. 1, Jan. 2005.

[4] N. Engheta and R. W. Ziolkowski, "A positive future for double-negative metamaterials," IEEE Trans. Microw. Theory Tech., vol. 53, no. 4, pp. 1535-1556, Apr. 2005.

[5] J. D. Joannopoulos, P. R. Villeneuve, and S. Fan, "Photonic crystals: Putting a new twist on light," Nature, vol. 386, no. 6621, pp. 143-149, Mar. 1997.

[6] J. B. Pendry, "Negative reflection makes a perfect lens," Phys. Rev. Lett., vol. 85, no. 18, pp. 3966-3969, Oct. 2000.

[7] L. Zhang, J. A. Castaneda, and N. G. Alexopoulos, "Scan blindness free phased array design using PBG materials," IEEE Trans. Antennas Propag., vol. 52, no. 8, pp. 2000-2007, Aug. 2004.

[8] S. Kawakami, "Analytically solvable model of photonic crystal structures and novel phenomena," J. Lightw. Technol., vol. 20, no. 8, pp. 1644-1650, Aug. 2002.

[9] T. Fujisawa and M. Koshiba, "Time-domain beam propagation method for nonlinear optical propagation analysis and its application to photonic crystal circuits," J. Lightw. Technol., vol. 22, no. 2, pp. 684-691, Feb. 2004

[10] T. Fujisawa and M. Koshiba, "Finite-element mode-solver for nonlinear periodic optical waveguides and its application to photonic crystal circuits," J. Lightw. Technol., vol. 23, no. 1, pp. 382-387, Jan. 2005.

[11] A. Taflove, Computational Electrodynamics. Norwood, MA: Artech House, 1995.

[12] F. Capolino, D. R. Jackson, and D. R. Wilton, "Fundamental properties of the field at the interface between air and a periodic artificial material excited by a line source," IEEE Trans. Antennas Propag., vol. 53, no. 1, pp. 91-99, Jan. 2005.

[13] D. J. Bergman, "The dielectric constant of a simple cubic array of identical spheres," J. Phys. C, Solid-State Phys., vol. 12, no. 22, pp. 4947-4960, Nov. 1979.
[14] D. J. Bergman and K.-J. Dunn, "Bulk effective dielectric constant of a composite with a periodic microgeometry," Phys. Rev. B, Condens. Matter, vol. 45, no. 23, pp. 13 262-13 271, Jun. 1992.

[15] J. B. Pendry, A. J. Holden, D. J. Robbins, and W. J. Steward, "Magnetism from conductors and enhanced nonlinear phenomena," IEEE Trans. Microw. Theory Tech., vol. 47, no. 11, pp. 2075-2084, Nov. 1999.

[16] D. R. Smith, D. C. Vier, N. Krol, and S. Schultz, "Direct calculation of permeability and permittivity for a left-handed metamaterial," Appl. Phys. Lett., vol. 77, no. 14, pp. 2246-2248, Oct. 2000.

[17] D. R. Smith and J. B. Pendry, "Homogenization of metamaterials by field averaging," J. Opt. Soc. Amer. B, Opt. Phys., vol. 23, no. 3, pp. 391-403, Mar. 2006.

[18] D. R. Smith, S. Shultz, P. Markos, and C. M. Soukoulis, "Determination of effective permittivity and permeability of metamaterials from reflection and transmission coefficients," Phys. Rev. B, Condens. Matter, vol. 65, no. 19, May 2002, 195104.

[19] T. Koschny, M. Kafesaki, E. N. Economou, and C. M. Soukoulis, "Effective medium theory of left-handed materials," Phys. Rev. Lett., vol. 93, no. 10 , Sep. $2004,107402$.

[20] X. Chen, T. M. Grzegorczyk, B. I. Wu, J. Pacheco, and J. A. Kong, "Robust method to retrieve the constitutive parameters of metamaterials," Phys. Rev. E, Stat. Phys. Plasmas Fluids Relat. Interdiscip. Top., vol. 70, Jul. 2004, 016608.

[21] D. R. Smith, D. C. Vier, T. Koschny, and C. M. Soukoulis, "Electromagnetic parameter retrieval from inhomogeneous metamaterials," Phys. Rev. E, Stat. Phys. Plasmas Fluids Relat. Interdiscip. Top., vol. 71, no. 3, Mar. 2005, 036617.

[22] N. Kinayman and M. I. Aksun, Modern Microwave Circuits. Norwood, MA: Artech House, 2005

[23] Y. Hua and T. K. Sarkar, "Generalized pencil-of-function method for extracting poles of an EM system from its transient response," IEEE Trans. Antennas Propag., vol. 37, no. 2, pp. 229-234, Feb. 1989.

[24] K. M. Leung and Y. F. Liu, "Full vector wave calculation of photonic band structures in face-centered-cubic dielectric media," Phys. Rev. Lett., vol. 65, no. 21, pp. 2646-2649, Nov. 1990.

[25] H. S. Sözüer, J. W. Haus, and R. Inguva, "Photonic bands: Convergence problems with the plane-wave method," Phys. Rev. B, Condens. Matter, vol. 45, no. 24, pp. 13962-13 972, Jun. 1992.

[26] S. Datta, C. T. Chan, K. M. Ho, and C. M. Soukoulis, "Effective dielectric constant of periodic structures," Phys. Rev. B, Condens Matter, vol. 48, no. 20, pp. 14936-14943, Nov. 1993.

[27] D.-Y. Jeong, Y. H. Ye, and Q. M. Zhang, "Effective optical properties associated with wave propagation in photonic crystals of finite length along the propagation direction," J. Appl. Phys., vol. 92, no. 8, pp. 4194-4200, Oct. 2002.

[28] T. A. Birks, J. C. Knight, and P. S. J. Russell, "Endlessly single-mode photonic crystal fiber," Opt. Lett., vol. 22, pp. 961-963, Jul. 1997.

[29] J. D. Joannopoulos, R. D. Meade, and J. N. Winn, Photonic Crystals: Molding the Flow of Light. Princeton, NJ: Princeton Univ. Press, 1995.

[30] J. B. Pendry, A. J. Holden, W. J. Stewart, and I. Youngs, "Extremely low frequency plasmons in metallic mesostractures," Phys. Rev. Lett., vol. 76, no. 25, pp. 4773-4776, Jun. 1996.

[31] R. W. Ziolkowski, "Design, fabrication and testing of double negative metamaterials," IEEE Trans. Antennas Propag., vol. 51, no. 7, pp. 1516-1529, Jul. 2003.

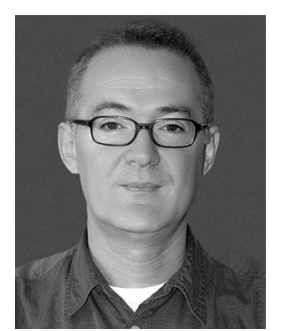

M. I. Aksun (M'92-SM'99) received the B.S. and M.S. degrees in electrical and electronics engineering from Middle East Technical University, Ankara, Turkey, in 1981 and 1983, respectively, and the Ph.D. degree in electrical and computer engineering from the University of Illinois at Urbana-Champaign, in 1990.

From 1990 to 1992, he was a Post-Doctoral Fellow with the Electromagnetic Communication Laboratory, University of Illinois at Urbana-Champaign. From 1992 to 2001, he was a member of the Department of Electrical and Electronics Engineering, Bilkent University, Ankara, Turkey, and became a Professor in 1999. In 2001, he joined the Department of Electrical and Electronics Engineering at Koç University, Istanbul, Turkey, as a Professor. In May 2004, he became the Dean of the Engineering College, Koç University. His recent research interests include numerical methods for electromagnetics and optics, printed circuits and antennas, and nanophotonics. 


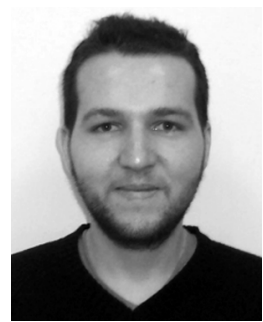

Aytaç Alparslan (S'04) received the B.S. degree in electrical and electronics engineering from Koç University, Istanbul, Turkey, in 2006, and is currently working toward the M.S. degree at Koç University.

$\mathrm{He}$ is currently a Research Assistant with the Microwaves and Antennas Research Laboratory, Koç University. His research interests include computational electromagnetics, metamaterials and photonic-bandgap structures, and nanophotonics.

Mr. Alparslan was the recipient of the Undergraduate Scholarship presented by the IEEE Microwave Theory and Techniques Society (IEEE MTT-S) and the IEEE Antennas and Propagation Society (IEEE AP-S) in 2005.

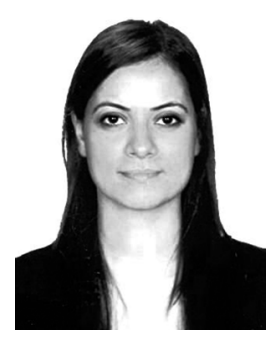

E. P. Karabulut (S'06) received the B.S. degree in electrical and electronics engineering from Middle East Technical University, Ankara, Turkey, in 2004, the M.S. degree in electrical and computer engineering from Koç University, Istanbul, Turkey, in 2006, and is currently working toward the Ph.D. degree in electrical and computer engineering at Koç University.

She is currently a Research Assistant with Koç University. Her research interests include numerical methods for electromagnetics and optics, computational aspects of periodic structures including bandgap materials and metamaterials, and nanooptics.

Ms. Karabulut was the recipient of the TUBITAK Ph.D. Scholarship.

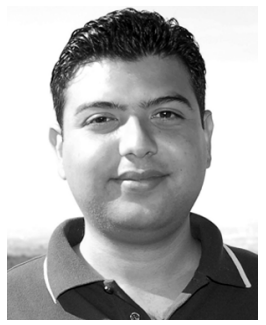

Erdinc Irci (S'06) was born on February 10, 1982, in Eskisehir, Turkey. He received the B.S. and M.S. degrees in electrical and electronics engineering from Bilkent University, Ankara, Turkey, in 2004 and 2007, respectively, and is currently working toward the Ph.D. degree at The Ohio State University, Columbus.

$\mathrm{He}$ is currently a Graduate Research Associate with the ElectroScience Laboratory, The Ohio State University. His research interests include EM theory, computational electromagnetics, antennas, scattering, metamaterials and photonic crystals.

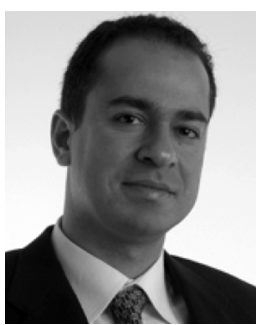

Vakur B. Ertürk (M'00) received the B.S. degree in electrical and electronics engineering from Middle East Technical University, Ankara, Turkey, in 1993, and the M.S. and Ph.D. degrees in electrical engineering from The Ohio State University (OSU), Columbus, in 1996 and 2000, respectively.

$\mathrm{He}$ is currently an Assistant Professor with the Electrical and Electronics Engineering Department, Bilkent University, Ankara, Turkey. His research interests include the analysis and design of planar and conformal arrays, active integrated antennas, scattering from and propagation over large terrain profiles, as well as metamaterials.

Dr. Ertürk served as the secretary/treasurer of the IEEE Turkey Section, as well as the Turkey Chapter of the IEEE Antennas and Propagation Society (IEEE AP-S), the IEEE Microwave Theory and Techniques Society (IEEE MTT-S), the Electron Devices Society, and the IEEE Electromagnetic Compatibility Society. He was the recipient of the 2005 URSI Young Scientist and 2007 Turkish Academy of Sciences Distinguished Young Scientist Awards. 